\title{
Universal scattering response across the type-II Weyl semimetal phase diagram
}

\author{
P. Rüßmann, ${ }^{1}$ A. P. Weber, ${ }^{2,3}$ F. Glott, ${ }^{4}$ N. Xu ${ }^{2,3}$ M. Fanciulli, ${ }^{2,3}$ S. Muff,,${ }^{2,3}$ A. Magrez, ${ }^{2}$ P. Bugnon, ${ }^{2}$ H. Berger, ${ }^{2}$ M. Bode, ${ }^{4}$ \\ J. H. Dil ${ }^{2,3}$ S. Blügel, ${ }^{1}$ P. Mavropoulos, ${ }^{1}$ and P. Sessi ${ }^{4, *}$ \\ ${ }^{1}$ Peter Grünberg Institut and Institute for Advanced Simulation, Forschungszentrum Jülich and JARA, 52425 Jülich, Germany \\ ${ }^{2}$ Institute of Physics, École Polytechnique Fédérale de Lausanne, 1015 Lausanne, Switzerland \\ ${ }^{3}$ Swiss Light Source, Paul Scherrer Institute, 5232 Villigen-PSI, Switzerland \\ ${ }^{4}$ Physikalisches Institut, Experimentelle Physik II, Universität Würzburg, Am Hubland, D-97074 Würzburg, Germany
}

(Received 10 April 2017; revised manuscript received 26 November 2017; published 5 February 2018)

\begin{abstract}
The discovery of Weyl semimetals represents a significant advance in topological band theory. They paradigmatically enlarged the classification of topological materials to gapless systems while simultaneously providing experimental evidence for the long-sought Weyl fermions. Beyond fundamental relevance, their high mobility, strong magnetoresistance, and the possible existence of even more exotic effects, such as the chiral anomaly, make Weyl semimetals a promising platform to develop radically new technology. Fully exploiting their potential requires going beyond the mere identification of materials and calls for a detailed characterization of their functional response, which is severely complicated by the coexistence of surface- and bulk-derived topologically protected quasiparticles, i.e., Fermi arcs and Weyl points, respectively. Here, we focus on the type-II Weyl semimetal class in which we find a stoichiometry-dependent phase transition from a trivial to a nontrivial regime. By exploring the two extreme cases of the phase diagram, we demonstrate the existence of a universal response of both surface and bulk states to perturbations. We show that quasiparticle interference patterns originate from scattering events among surface arcs. Analysis reveals that topologically nontrivial contributions are strongly suppressed by spin texture. We also show that scattering at localized impurities can generate defect-induced quasiparticles sitting close to the Weyl point energy. These give rise to strong peaks in the local density of states, which lift the Weyl node, significantly altering the pristine low-energy spectrum. Remarkably, by comparing the $\mathrm{WTe}_{2}$ and the $\mathrm{MoTe}_{2}$ cases we found that scattering response and topological transition are not directly linked. Visualizing the existence of a universal microscopic response to scattering has important consequences for understanding the unusual transport properties of this class of materials. Overall, our observations provide a unifying picture of the type-II Weyl phase diagram.
\end{abstract}

DOI: 10.1103/PhysRevB.97.075106

\section{INTRODUCTION}

The discovery of topological insulators (TIs) [1,2] led to intense research efforts in searching for materials whose properties are determined by band structure topology. In this context, the recent discovery of Weyl semimetals represents a milestone $[3,4]$. Contrary to TIs, where topological properties are manifested only at boundaries as gapless Dirac states, Weyl semimetals host bulk as well as surface topologically protected quasiparticles. In particular, Weyl points appear at crossing points that are protected by the topology of the bulk band structure. Because of the well-known surface-tobulk correspondence, they are necessarily associated with the appearance of new topologically protected boundary modes at the surface, the so-called topological Fermi arcs, which occupy unclosed Fermi contours connecting Weyl points of opposite chirality $[5,6]$.

Beyond their fundamental relevance, Weyl semimetals are characterized by intriguing transport properties. Very high mobility, extremely strong magnetoresistance [7], and even more exotic phenomena such as the chiral anomaly [8] or the

\footnotetext{
*Corresponding author: sessi@physik.uni-wuerzburg.de
}

possibility for Fermi arcs to tunnel through the bulk via the Weyl points have been discussed [9]. Because of the robustness inherited from topological protection, these effects are raising great expectations for direct applications of these materials in spintronics and magnetoelectrics.

Weyl semimetals were first experimentally discovered in the TaAs monopnictide family, known as type I [3,4,10-14]. More recently, it has been suggested that once Lorentz invariance is broken, a new flavor of Weyl material can be realized, the so-called type II, where strongly tilted Weyl cones emerge at the boundaries between bulk electron and hole pockets [15]. Theoretical predictions identified the $\mathrm{Mo}_{x} \mathrm{~W}_{1-x} \mathrm{Te}_{2}$ family as promising compounds [15-18]. One of the most interesting aspects of type-II Weyl materials is the possibility to scan their phase diagram passing the transition by continuously tuning their stoichiometry [18]. The resulting phase diagram offers an ideal platform to explore the functional response of the electronic properties and the existence of unifying trends within the Weyl phase.

\section{THE TYPE-II WEYL SEMIMETAL PHASE DIAGRAM}

The $\mathrm{Mo}_{x} \mathrm{~W}_{1-x} \mathrm{Te}_{2}$ family is characterized by a layered structure which crystallizes in a $T_{d}$ orthorhombic cell lacking 


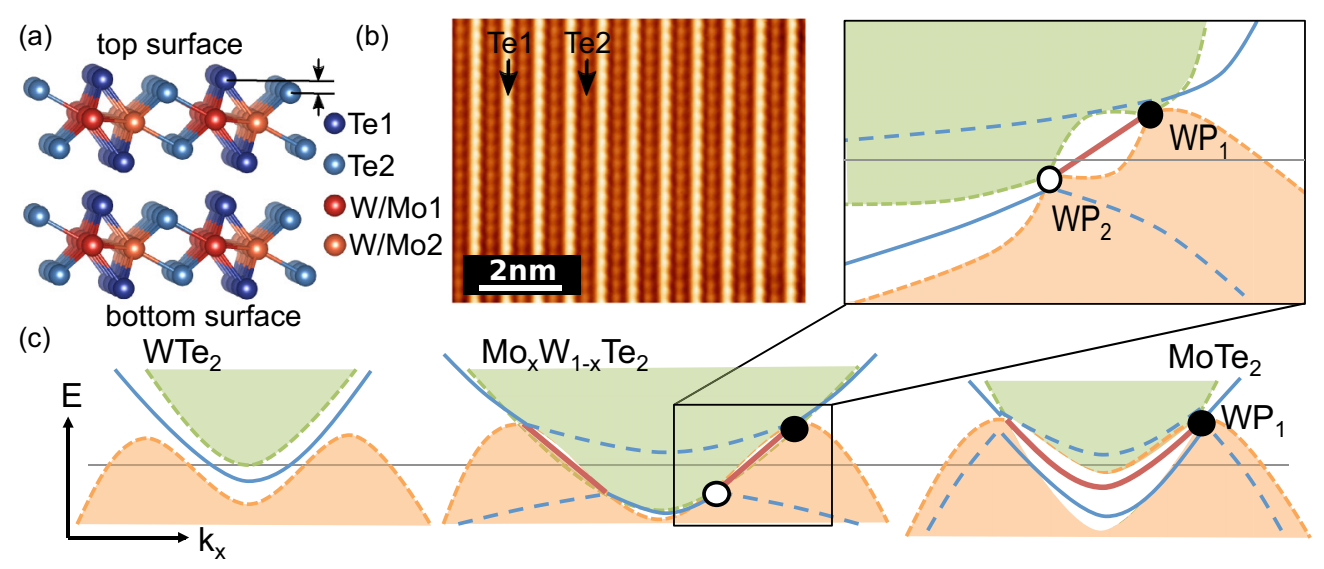

(d)

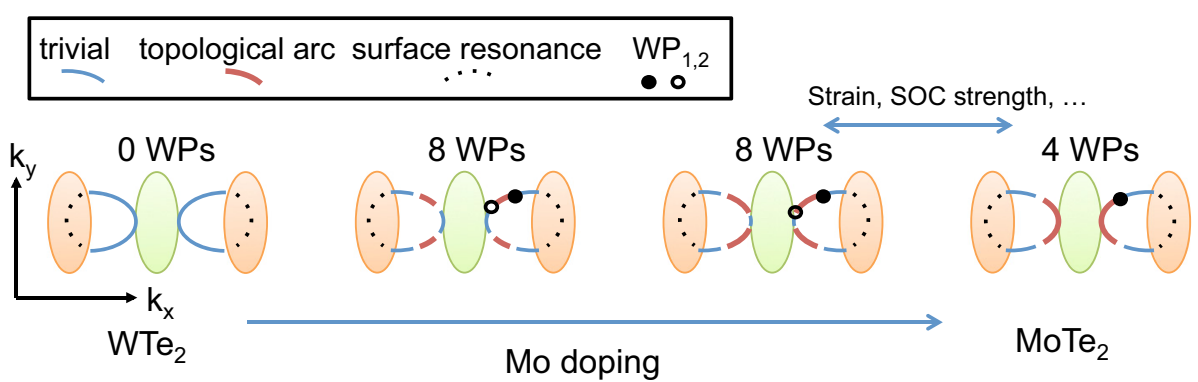

FIG. 1. (a) Crystal structure of $\mathrm{MoTe}_{2}$ and $\mathrm{WTe}_{2}$. (b) Atomically resolved STM topography of the WTe $\mathrm{W}_{2}$ surface. The existence of two inequivalent Te sites, labeled $\mathrm{Te} 1$ and $\mathrm{Te} 2$, with $\mathrm{Te} 1$ being slightly higher than $\mathrm{Te}$, gives rise to the columnlike character visible in the STM image. (c) Schematic representation of the type-II Weyl phase diagram. Although being topologically trivial, WTe $\mathrm{C}_{2}$ is found to lie very close to the topological phase transition and to host a trivial surface state (blue); small lattice distortions can easily drive it into a Weyl phase, with part of the surface state becoming topologically protected (red). By starting from $\mathrm{WTe}_{2}$ and substituting $\mathrm{W}$ with Mo, the system enters into a progressively more stable Weyl phase. (d) Evolution of the topological Fermi arcs as a function of the Mo concentration. By increasing the Mo concentration, topological Fermi arcs (red lines) become progressively larger.

inversion symmetry at low temperatures (space group $P m n 2_{1}$ ) [19]. The transition metal (W and Mo) planes are separated by Te bilayers, as shown in Fig. 1(a). Adjacent Te-(W/Mo)-Te trilayers are weakly bound by van der Waals forces, thus offering a natural cleaving plane. As a result, the surface exposed after cleaving is always Te terminated. As illustrated in Fig. 1(a), the Te atoms occupy two inequivalent sites, labeled $\mathrm{Te} 1$ and Te2, with one slightly protruding over the other. This is reflected in the columnlike character visible in the atomically resolved scanning tunnel microscope (STM) image reported in Fig. 1(b).

Density functional theory calculations indicated how, by starting from $\mathrm{WTe}_{2}$, which lies close to a topological phase transition, and substituting W with Mo, the system enters into a progressively more stable Weyl phase, as schematically illustrated in Fig. 1(c) [18]. In particular, by increasing the Mo concentration, the Weyl points become well separated in reciprocal space and thus cannot easily be annihilated by small perturbations [18]. The larger Weyl point separation has direct consequences for the surface, with topological Fermi arcs getting progressively larger [see red lines in Fig. 1(d)]. In this respect, it is worth noticing that the surface electronic structure of these compounds is substantially complicated by the concomitant existence of trivial surface states. Although they do not form open topological arcs, trivial states partially overlap with the projected bulk electronic structure, giving rise to surface resonances [dashed lines in Fig. 1(d)] which are characterized by a reduced surface spectral weight. As a result, their pure surface state part (blue line) can effectively mimic an open arclike contour.

Experimentally investigating this phase diagram proved problematic. This is mainly due to two reasons: (i) Contrary to type-I Weyl materials, in type II, the projection of the Weyl points onto the surface overlaps with several bulkderived trivial states, thereby complicating the discrimination of topological Fermi arcs from other states of trivial origin [20-27]. (ii) The Weyl points are expected to appear at energies above the Fermi level [15-18], where they are inaccessible to conventional photoemission techniques [20-27]. In this context, while a general consensus exists over the topological nature of the $\mathrm{MoTe}_{2}$ arcs [20-22], the situation appears highly controversial for $\mathrm{WTe}_{2}$. Although surface arcs have clearly been observed in several photoemission studies, their topological or trivial nature is highly debated, with different studies reaching conflicting conclusions [23-26]. Ab initio calculations also show that, while $\mathrm{MoTe}_{2}$ is well inside the Weyl regime, $\mathrm{WTe}_{2}$ is in close proximity to a Weyl phase transition [23]. This can give rise to Weyl points of opposite chirality which are very close in reciprocal space and thus can easily be annihilated by very small lattice distortions induced by strain or temperature, as discussed in Ref. [23]. Therefore, it is particularly important to investigate the existence of universal signatures spanning the entire Weyl phase diagram, e.g., by comparing the two extreme cases, $\mathrm{MoTe}_{2}$ and $\mathrm{WTe}_{2}$. 
(a)
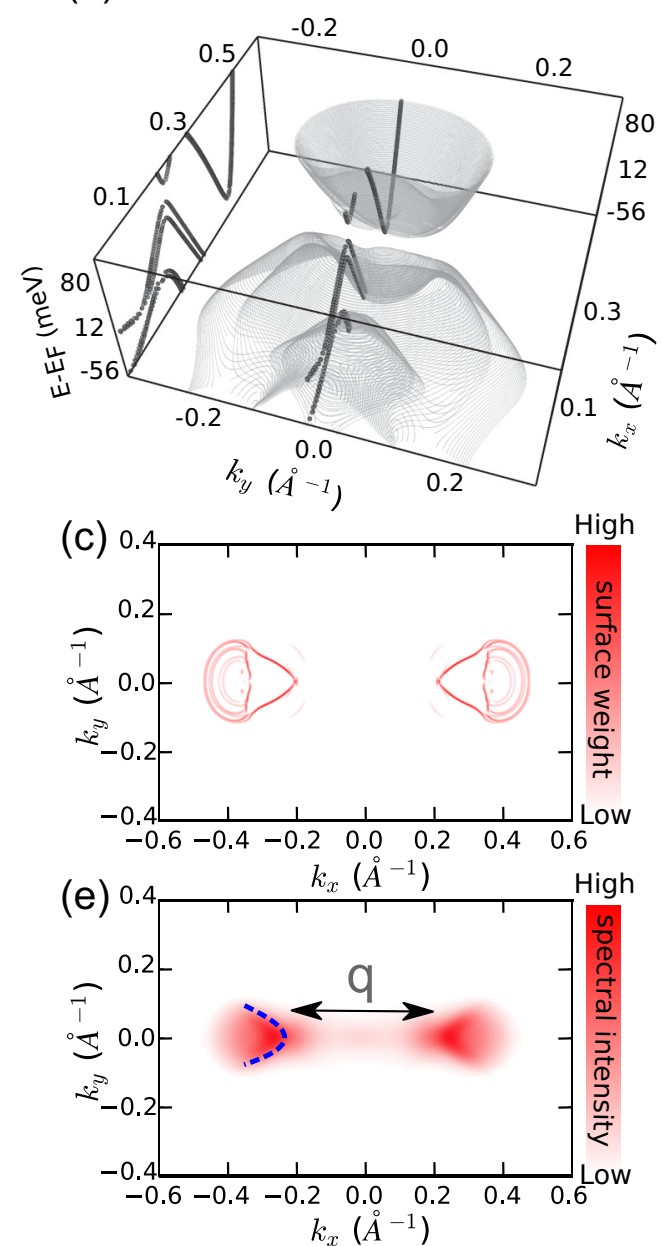

(b)

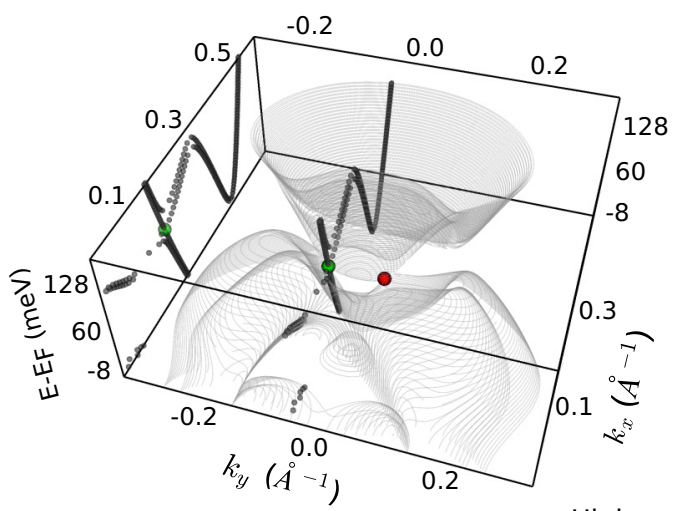

(d)

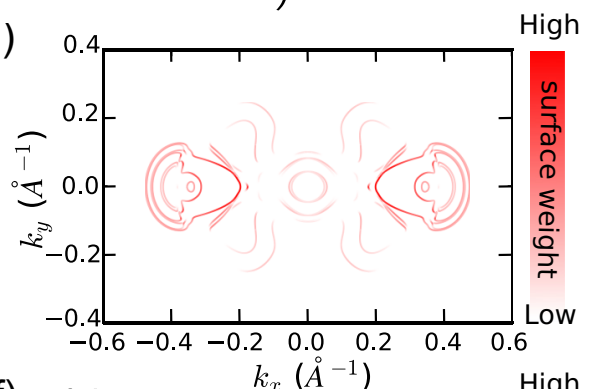

(f)

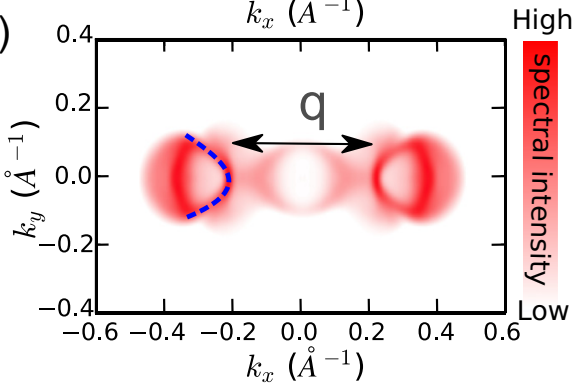

FIG. 2. (a) and (b) Calculated bulk band structure at $k_{z}=0$ and (c) and (d) theoretical and (e) and (f) experimental Fermi surface for WTe ${ }_{2}$ and $\mathrm{MoTe}_{2}$, respectively. In (a), the dotted lines show the band structure along $k_{y}$ at the particular $k_{x}$ where the electron and hole pockets approach each other the closest. In (b), the dotted lines show the band structure along $k_{y}$ at the specific $k_{x}$ where Weyl points appear (green and red circles, reflection symmetric around $k_{x}=0$ ). The experimental Fermi surfaces have been obtained with angle-resolved photoemission spectroscopy at temperature $T<60 \mathrm{~K}$ using photon energies of 20 and $24 \mathrm{eV}$ for $\mathrm{MoTe}_{2}$ and $\mathrm{WTe}_{2}$, respectively.

Here, we visualize the response of $\mathrm{MoTe}_{2}$ and $\mathrm{WTe}_{2}$ and discuss the results in terms of Weyl nodes and Fermi arcs. We identify the existence of a universal response of these systems to perturbations, which is found to be composition independent. In particular, we report the emergence of well-defined quasiparticle interference patterns originating from surface arcs. Contrary to earlier studies [22], we (i) can clearly resolve their open contour and (ii) demonstrate that contributions from Fermi arcs connecting Weyl points are strongly suppressed because of their spin texture, which protects them from backscattering $[22,28]$. Furthermore, in line with the theoretical predictions of the response of Weyl semimetals, we reveal the emergence of new quasiparticles arising at the Weyl point energy, which lift the density-of-states minimum associated with the Weyl node $[29,30]$.

\section{SURFACE SCATTERING: THE ROLE OF FERMI ARCS}

Figures 2(a) and 2(b) report the electronic band structure of bulk $\mathrm{WTe}_{2}$ and $\mathrm{MoTe}_{2}$ at $k_{z}=0$, respectively, where due to the crystal symmetry Weyl points are possible [15], calculated with the density-functional-based full-potential relativistic Korringa-Kohn-Rostoker Green's-function method [31-34]. Computational details can be found in the Supplemental Material [35]. The combined effects of the inversion asymmetry of the crystal structure and the strong spin-orbit coupling characterizing these materials give rise to a spin-polarized band structure [36]. However, whereas Weyl points are emerging in $\mathrm{MoTe}_{2}$ [see green and red dots in Fig. 2(b), which identify one pair of Weyl points of opposite chirality], a gap between electron and hole pockets is clearly visible for $\mathrm{WTe}_{2}$ [Fig. 2(a)], indicating the trivial character of this compound. In this context, it is worth noticing that the electronic structure of $\mathrm{WTe}_{2}$ is very delicate. As discussed in Ref. [18], small changes in the lattice constant can drive the system into a nontrivial state hosting eight Weyl points, proving the close vicinity of a topological phase transition. Despite these differences highlighted by band structure calculations, our angle-resolved photoemission data reported in Figs. 2(e) and 2(f) reveal the presence of arclike electronic structures in both compounds 

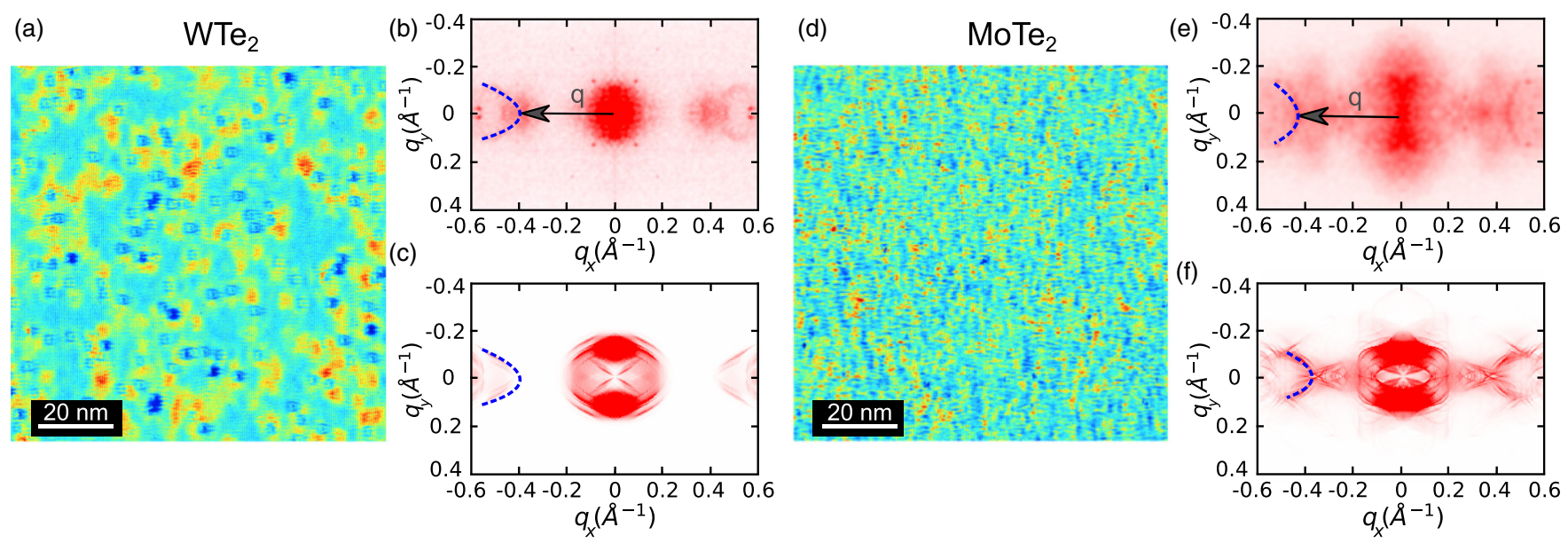

FIG. 3. (a) Differential conductance $d I / d U$ map close to the Fermi level ( $E-E_{\mathrm{F}}=10 \mathrm{meV}$ ); (b) Fourier-transformed $d I / d U$ map and (c) theoretically calculated quasiparticle interference pattern obtained on $\mathrm{WTe}_{2}$. (d) Differential conductance $d I / d U$ map close to the Fermi level $\left(E-E_{\mathrm{F}}=10 \mathrm{meV}\right)$; (e) Fourier-transformed $d I / d U$ map and (f) theoretically calculated quasiparticle interference pattern obtained on $\mathrm{MoTe}_{2}$. The quasiparticle interference pattern is dominated by scattering events between opposite Fermi arcs. Some additional sharp spots which do not have any correspondence in the theoretical panels are also visible [see, for example, (b) at $\mathbf{q}=(0.1,0.1) \AA^{-1}$ ]. They are due to experimental noise and thus do not have any importance for the following discussion.

(see dashed lines). An overall agreement between theory and experiments is found. Although it is tempting to assign the arclike features in Figs. 2(e) and 2(f) to topological Fermi arc states, comparison with calculated constant-energy contours reported in Figs. 2(c) and 2(d) reveals a more complicated scenario. Indeed, $a b$ initio calculations reveal that several electronic features coexist within a very small part of the first Brillouin zone which cannot fully be resolved by photoemission experiments (see Supplemental Material, Sec. B [35]). It is worth stressing that, as discussed above, an analysis of our calculations reveals that most of the arcs visible in Figs. 2(c) and 2(d) are of trivial origin and that topological Fermi arcs are also present only for $\mathrm{MoTe}_{2}$. This proves that the observation of surface arcs is not a sufficient condition to unequivocally imply the existence of Weyl points.

To gain insight in trivial vs topological Fermi arc contributions, we performed quasiparticle interference experiments. This technique makes use of the standing-wave pattern generated by elastic scattering of electronic states at surface defects and has been proven to be a powerful method to test the properties of topological materials [37-42]. Contrary to conventional photoemission spectroscopy, it gives access to both occupied and unoccupied electronic states, thereby providing a complete spectroscopic characterization of all relevant electronic features around the Fermi energy. This is particularly important for type-II Weyl materials, where Weyl points are theoretically expected to emerge above the Fermi level [15-18]. Figures 3(a) and 3(d) report differential conductance $d I / d U$ maps acquired in close proximity to the Fermi level $\left(E-E_{\mathrm{F}}=10 \mathrm{meV}\right)$ on $\mathrm{WTe}_{2}$ and $\mathrm{MoTe}_{2}$, respectively. Their Fourier transformations, reported in Figs. 3(b) and 3(e), allow us to conveniently analyze scattering channels in reciprocal space. Our results reveal the emergence of clear arclike interference patterns, as indicated by the black arrows in both compounds which develop along the $q_{x}$ direction at approximately $q_{x}=0.4 \AA^{-1}$ (see dashed blue lines). To shed light on the origin of these vectors, experimental data have been compared with calculated interference patterns. Our theoretical approach employs the extended joint density of states (JDOS) model [43], with the impurity electronic structure and scattering amplitude calculated from the self-consistent potential by means of the Korringa-Kohn-Rostoker Green'sfunction method and with the contribution of scattering vectors being weighted according to the backscattering condition that promotes standing waves in scanning tunneling spectroscopy (STS) (for details, see Ref. [35]). We considered a number of plausible intrinsic impurities [35]. Searching for the ones that reproduce in the calculation the measured resonance position and the qualitative form of the quasiparticle interference (QPI), we arrived at $\mathrm{Mo}$ (W in $\mathrm{WTe}_{2}$ ) antisites substituting surface Te atoms. As we found, possible spin polarization of impurities has a negligible effect on the QPI spectra, even though they formally open the time-reverse scattering channel [35]. Results for $\mathrm{WTe}_{2}$ and $\mathrm{MoTe}_{2}$ are reported in Figs. 3(c) and 3(f). As for the experimental results, an arclike feature is clearly visible in both cases along the $q_{x}$ direction (see dashed lines), which originates from interarc scattering among opposite Fermi arcs. This assignment is further supported by direct comparison with photoemission data [see Figs. 2(e) and 2(f), where the scattering vector $q$ corresponds to the distance connecting opposite arcs].

Indeed, Fourier-transformed quasiparticle interference peaks at $\mathbf{q}=\mathbf{k}_{f}-\mathbf{k}_{i}$, where $\mathbf{k}_{i}$ and $\mathbf{k}_{f}$ are the wave vectors of initial and final states with opposite group velocities supporting constructive interference and $\mathbf{q}$ is the scattering vector connecting them. A quantitative comparison with the experimental constant-energy contours reported in Figs. 2(e) and 2(f) allows us to directly link these interference phenomena to interarc scattering among opposite Fermi arcs. Furthermore, contrary to photoemission data, the higher surface sensitivity of the STM unequivocally shows the contour character of the arcs. Especially in Fig. 3(b), the arc starting at $\mathbf{q}=(0.4,0) \AA^{-1}$ 


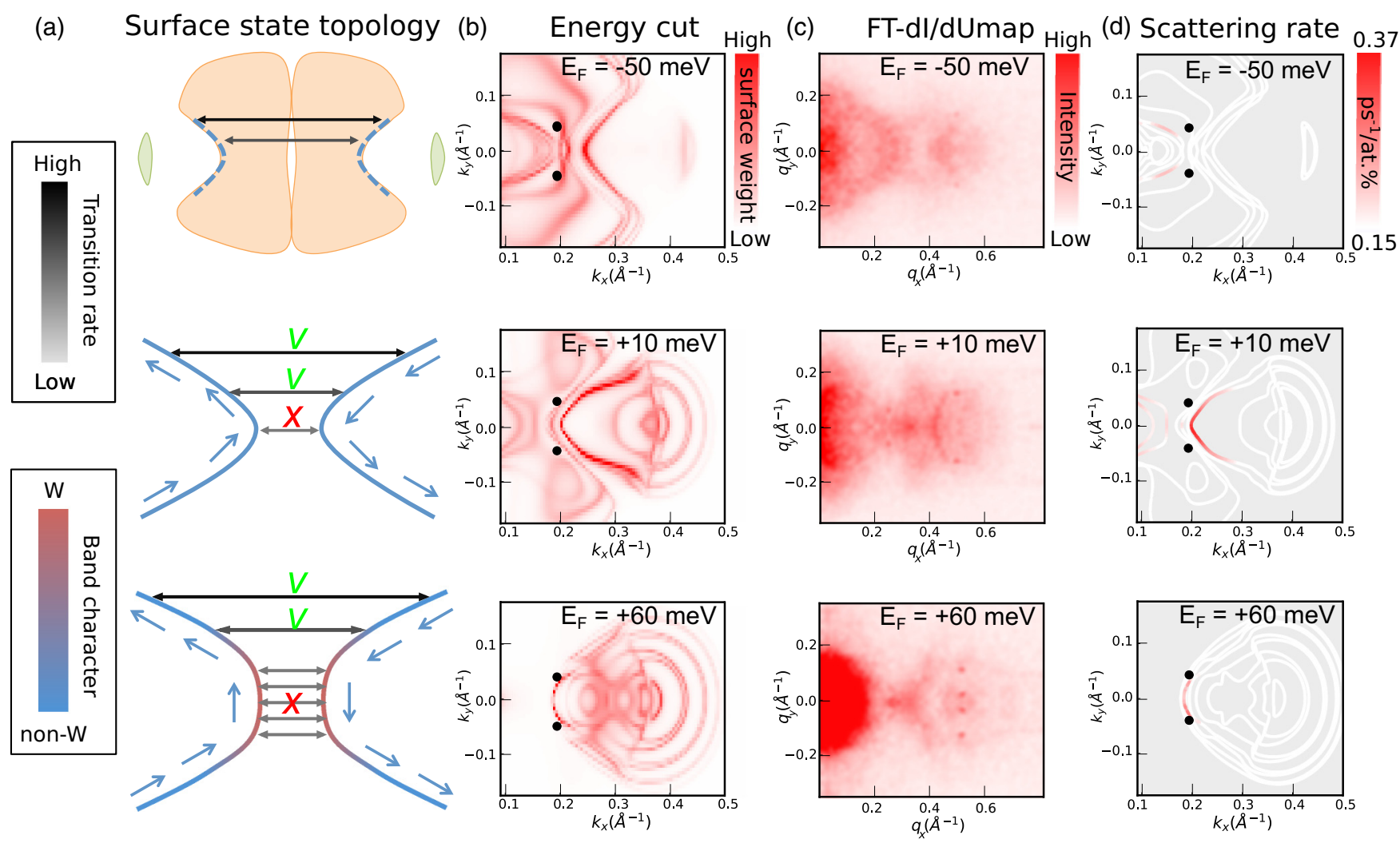

FIG. 4. (a) Schematic representation of the scattering events among Fermi arcs in $\mathrm{MoTe}_{2}$. The band character color code changes from red (labeled "W") in parts of the constant energy contour that are in the proximity of the topological surface arc connecting the Weyl points at $E_{\mathrm{F}}+60 \mathrm{meV}$ to blue (labeled "non-W") in parts of the contour or energies farther away from the Weyl points. (b) Theoretically calculated surface-projected constant energy cuts. (c) Experimentally obtained Fourier-transformed quasiparticle interference patterns. (d) Theoretically calculated scattering rate. In (b) and (d) the black dots identify the Weyl points. All results are given for three representative energies: below the Fermi level (top panels), close to the Fermi level (middle panels), and close to the position of the Weyl points, where the extension of the topologically nontrivial arcs is maximized (bottom panels).

and extending to the right appears to be closing in a loop at $\mathbf{q}=(0.6,0) \AA^{-1}$. We believe that this is because of two distinct scattering channels. Looking at Fig. 2(c), the most surface localized states (most intensively drawn contours) form a "nose" starting at $\mathbf{k}=(0.2,0) \AA^{-1}$ and ending at $\mathbf{k}=(0.3 \pm 0.1) \AA^{-1}$ and a "moustache" at $k_{x}=0.35 \AA^{-1}$, extending vertically between $k_{y}= \pm 0.1 \AA^{-1}$ (with symmetric images at $k_{x}<0$ ). Scattering vectors between the two noses on opposite sides of the Brillouin zone center compose the left half of the aforementioned closed loop in the QPI, while vectors between the nose and the mustache on opposite sides of the Brillouin zone center compose the right half of the closed loop. We also notice that the measured arc in Fig. 2(e) has a high intensity at $q_{y}=0$, while the corresponding calculation in Fig. 2(f) shows a white spot (zero intensity) at $q_{y}=0$. We believe that this is because of the limitations in experimental resolution; indeed, next to the white spot and in the direction of the Brillouin zone center in Fig. 2(f) a band crossing with high intensity appears that could be contributing to the arc in the experimental figure due to the finite resolution.

In this context, it is worth noticing that the close proximity of $\mathrm{WTe}_{2}$ to a Weyl transition allows one to safely exclude any significant contribution of topological Fermi arcs to the observed interference patters for this compound. Even when considering a slightly distorted structure hosting Weyl points, they would be so close in reciprocal space that the extension of topological Fermi arcs connecting them would be negligible. This is not the case for $\mathrm{MoTe}_{2}$, where, by progressively moving towards the energy position of the Weyl points, topologically nontrivial Fermi arcs span a much larger fraction of the Brillouin zone. This is illustrated by the schematic representation reported in Fig. 4(a) and the theoretically calculated constant energy cuts displayed in Fig. 4(b). At low energies ( $E_{\mathrm{F}}-50 \mathrm{meV}$, top), the surface states are absorbed in the bulk bands, forming surface resonances and keeping the surface localization relatively low. Thus the impurity scattering [Fig. 4(d)] and the QPI signal [Fig. 4(c)] are weak. At higher energies $\left(E_{\mathrm{F}}+10 \mathrm{meV}\right.$, middle $)$ there is a formation of arcs that are genuine surface states and cover a comparatively larger portion of the surface Brillouin zone. As a result, a well-defined arclike interference pattern appears in Fig. 4(c), caused by strong interarc scattering. Evidence for this is shown in the middle panel of Fig. 4(d), where the scattering rate of states in the surface arc is high. Finally, as the Weyl point energy is approached $\left(E_{\mathrm{F}}+60 \mathrm{meV}\right.$, bottom), an arc is found connecting the surface-projected Weyl points [their positions are shown by black dots in Figs. 4(b) and 4(d)]. The arc is illustrated as the red part of the line in Fig. 4(a), but it is also clear in Fig. 4(d), where it is practically the only part in the band structure that suffers 
(a)

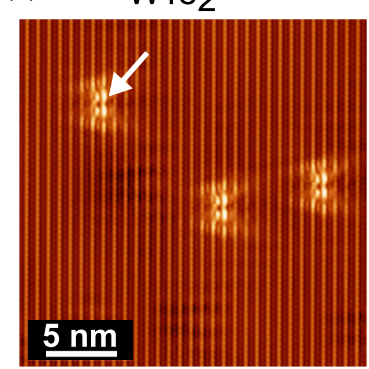

(b)

(b) $\quad$ MoTe2

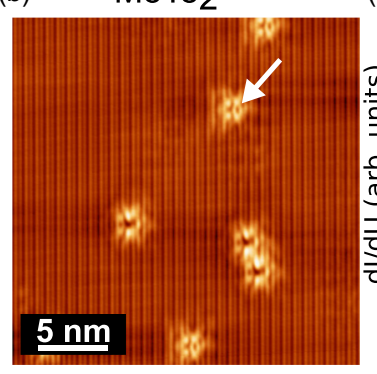

(c)

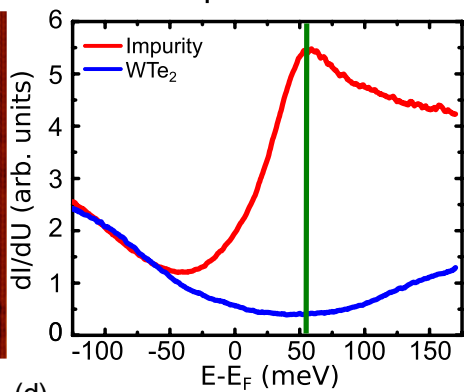

(d)

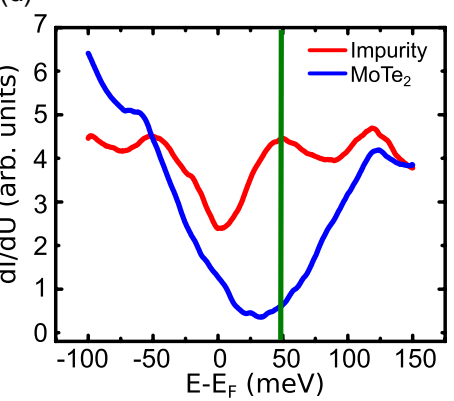

(e)

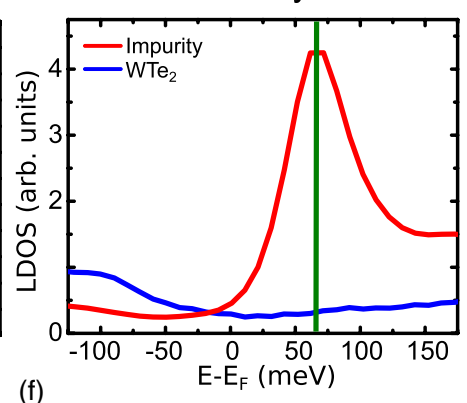

(f)

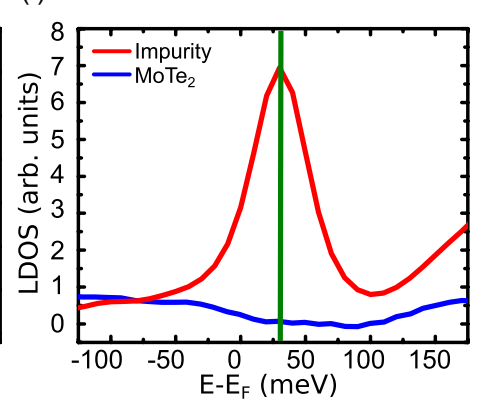

FIG. 5. (a) and (b) Topographic images acquired for $\mathrm{WTe}_{2}$ and $\mathrm{MoTe}_{2}$. In both cases, intrinsic defects are present on the surface. (c) and (d) Comparison of scanning tunneling spectroscopy data taken in a defect-free area (blue line) and by positioning the tip on top of antisite defects (W and Mo substituting Te), revealing the emergence of quasiparticle resonances close the Weyl point energy (see discussion in the text). (e) and (f) Ab initio calculated local densities of states on the pristine surface (blue line) and on top of an antisite defect (red line) confirm the experimental findings. Green vertical lines are used to identify the position of the peak maximum.

impurity scattering. Here, the arc is considerably flattened, providing nesting conditions for interarc scattering and a strong QPI signal. However, the spin polarization in the two opposite arcs points in opposite directions. Thus the spin texture results in an effective protection against interarc scattering, weakening the QPI signal in Fig. 4(c). Such behavior is reminiscent of the forbidden backscattering originally discussed for Rashba systems [44] and, more recently, for topological insulators [38]. In the present case, however, the presence of large parallel segments with opposite spin polarization significantly extend the protection well beyond time-reversal-symmetry partner states.

We should note that, while Fig. 4(d) depicts the scattering rate of each initial state integrated over all final states (including interarc and intra-arc scattering), we also calculated the scattering rate $\left\langle P_{\mathbf{k k}^{\prime}}\right\rangle$ averaged over initial and final surfacelocalized states on opposite arcs. It is approximately 5 times stronger in the middle panel $\left(E_{\mathrm{F}}+10 \mathrm{meV}\right)$ than in the bottom panel $\left(E_{\mathrm{F}}+60 \mathrm{meV}\right)$, explaining the weaker Fouriertransformed QPI intensity in the latter case. In addition, our calculations show that spin-polarized Mo impurities change the scattering intensity by less than $5 \%$; that is, in practice they do not open a significant backscattering channel; thus their contribution to the Fourier-transformed QPI is almost the same as that of nonmagnetic impurities (see the extended JDOS images in Ref. [35]).

\section{BULK SCATTERING: THE ROLE OF WEYL POINTS}

Finally, we discuss the effect impurities have on Weyl nodes. Recent theoretical predictions showed that a common characteristic of Dirac-like materials is the emergence of impurityinduced quasiparticles which lift the Dirac node [30]. This behavior has been recently confirmed in topological insulators, where impurity resonances induced by magnetic dopants have been shown to effectively fill the gap which is expected to open at the Dirac point in magnetically ordered samples [45]. A similar behavior has been proposed to arise in Weyl semimetals. In particular, scattering at localized impurities is expected to lift the Weyl node by inducing new quasiparticle resonances close to the Weyl point energy [29,30]. The emergence of these quasiparticles has been theoretically proposed as a signature of a Weyl phase. This has been experimentally investigated in Figs. 5(a) and 5(b), which show topographic images acquired for the two different compounds, i.e., $\mathrm{WTe}_{2}$ and $\mathrm{MoTe}_{2}$. Intrinsic defects highlighted by arrows are visible in both cases. They have been identified as antisites (W or Mo atoms substitute Te in the topmost layer) and are commonly found in transition-metal dichalcogenides [46-48]. Figures 5(c) and 5(d) show STS data acquired for both materials by positioning the tip on top of (red line) and far away from (blue line) a defect. The minimum, visible in proximity to the Fermi level in defect-free areas, highlights the semimetallic character of these compounds. However, on top of defects a strong peak is visible in both materials which lifts the local-density-of-states minimum.

These defect-induced quasiparticle resonances appear very close to the energy where Weyl points are expected to emerge (see vertical green line which highlights the peak maximum). The experimental findings are supported by the $a b$ initio-calculated local density of states at the impurity atom. Considering that the resonance must develop from $d$ states of a transition-metal surface defect, we find excellent agreement of the resonance position between calculations and experiment in the case of a nonmagnetic Mo impurity in the 
Te1 surface position [Fig. 5(e)] or a magnetic Mo impurity in the $\mathrm{Te} 2$ surface position for $\mathrm{MoTe}_{2}$ (density of states shown in Ref. [35]). As we explain in Ref. [35], both scenarios are plausible. From the absence of a Kondo resonance at $E_{\mathrm{F}}$ in the STS [49], we can exclude the scenario in which the magnetic moments are screened by fluctuations that may occur in Weyl semimetals [50]. For $\mathrm{WTe}_{2}$, we also find excellent agreement with a nonmagnetic $\mathrm{W}$ impurity in the Te2 surface position [Fig. 5(f)]. More precisely, in $\mathrm{MoTe}_{2}$ the experimentally observed impurity-induced quasiparticle resonance is positioned at the calculated Weyl point energy $\left(E_{\mathrm{F}}+48\right.$ $\mathrm{meV})$. As discussed above, for $\mathrm{WTe}_{2}$ our calculations predict a trivial material near a topological phase transition into a Weyl phase. In this case, the impurity-induced resonances emerge energetically close to the very narrow gap in $\mathbf{k}$ space between electron and hole pockets, where Weyl points are expected to emerge according to Refs. $[15,23,26]$. Our calculations suggest that the resonances are not necessitated by the Weyl points but may be trivially due to $d$ states hybridizing with a metallic environment.

\section{CONCLUSIONS AND DISCUSSION}

Our observations provide strong evidence that topological Weyl transitions are accompanied by continuous, smooth transitions of electronic structure observables that are frequently regarded as hallmarks of the topological state: Polarized surface states and resonances close to the Weyl point energy occur on each side of the transition. It follows that, although topological indices change when driving the system through a topological quantum phase transition, important band-structuredependent experimental observables, such as the impurityinduced resonances and spin-polarized surface states reported here, are not characterized by any discontinuity, i.e., on-off behavior. It may well be that the continuity originates in the similarity of the materials on the two sides of the transitions and that for this reason the trivial surface states and resonances of $\mathrm{WTe}_{2}$ transform into topologically preconditioned quantities in $\mathrm{MoTe}_{2}$. Still, experimental observations of arclike surface states or resonances close to candidate Weyl points are clearly insufficient evidence for the Weyl phase.
We would like to stress that the relevance of our observations goes well beyond topological band structure aspects. It is well known that defects and disorder can significantly impact transport properties, and their presence has been suggested to be at the origin of both positive and negative magnetoresistance effects [51,52]. In this context, our observations contribute by providing a detailed microscopic picture of the resonant scattering at impurities in type-II Weyl semimetals. In particular, we demonstrate that intrinsic defects significantly alter the local density of states close to the Weyl points, ultimately changing the low-energy spectrum of Weyl semimetals. We conclude that the presence of defects cannot be overstressed and suggest that they play an important role in determining the fascinating transport properties of this class of materials $[53,54]$ on either side of the transition in the Weyl phase diagram.

Overall, we reveal a response to surface perturbations that holds universally across the type-II Weyl semimetal phase diagram. We show that surface arcs dominate the quasiparticle interference pattern, with the Fermi arc contribution at the Weyl point energy being strongly suppressed by its spin texture. In agreement with theoretical predictions, we also demonstrate that impurity-induced quasiparticle resonances can emerge close to the energy where Weyl points are expected. Our observations highlight that the functional response of both surface and bulk states to perturbations in this class of materials does not depend on whether we have passed the Weyl phase transition or we are simply close to it. This allows us to infer the existence of a stoichiometry-independent response to perturbations for type-II Weyl semimetals, providing a unifying picture of the type-II Weyl phase diagram.

Note added in proof: Recently, we learned of a related work on the $\mathrm{WTe}_{2}$ surface [69].

\section{ACKNOWLEDGMENTS}

This work was supported by the Deutsche Forschungsgemeinschaft within SPP 1666 (Grants No. BO1468/21-1 and No. MA4637/3-1) and through SFB 1170 "ToCoTronics" (project A02). P.R., P.M., and S.B. acknowledge financial support from the VITI project of the Helmholtz Association as well as computational support from the JARA-HPC Supercomputing Centre at RWTH Aachen University.
[1] M. Z. Hasan and C. L. Kane, Rev. Mod. Phys. 82, 3045 (2010).

[2] X.-L. Qi and S.-C. Zhang, Rev. Mod. Phys. 83, 1057 (2011).

[3] S.-Y. Xu, I. Belopolski, N. Alidoust, M. Neupane, G. Bian, C. Zhang, R. Sankar, G. Chang, Z. Yuan, C.-C. Lee et al., Science 349, 613 (2015).

[4] B. Q. Lv, H. M. Weng, B. B. Fu, X. P. Wang, H. Miao, J. Ma, P. Richard, X. C. Huang, L. X. Zhao, G. F. Chen et al., Phys. Rev. X 5, 031013 (2015).

[5] H. Weng, C. Fang, Z. Fang, B. A. Bernevig, and X. Dai, Phys. Rev. X 5, 011029 (2015).

[6] S.-M. Huang, S.-Y. Xu, I. Belopolski, C.-C. Lee, G. Chang, B. Wang, N. Alidoust, G. Bian, M. Neupane, C. Zhang et al., Nat. Commun. 6, 7373 (2015).
[7] C. Shekhar, A. K. Nayak, Y. Sun, M. Schmidt, M. Nicklas, I. Leermakers, U. Zeitler, Y. Skourski, J. Wosnitza, Z. Liu et al., Nat. Phys. 11, 645 (2015).

[8] X. Huang, L. Zhao, Y. Long, P. Wang, D. Chen, Z. Yang, H. Liang, M. Xue, H. Weng, Z. Fang et al., Phys. Rev. X 5, 031023 (2015).

[9] A. C. Potter, I. Kimchi, and A. Vishwanath, Nat. Commun. 5, 5161 (2014).

[10] L. X. Yang, Z. K. Liu, Y. Sun, H. Peng, H. F. Yang, T. Zhang, B. Zhou, Y. Zhang, Y. F. Guo, M. Rahn et al., Nat. Phys. 11, 728 (2015).

[11] S.-Y. Xu, N. Alidoust, I. Belopolski, Z. Yuan, G. Bian, T.-R. Chang, H. Zheng, V. N. Strocov, D. S. Sanchez, G. Chang et al., Nat. Phys. 11, 748 (2015). 
[12] Z. K. Liu, L. X. Yang, Y. Sun, T. Zhang, H. Peng, H. F. Yang, C. Chen, Y. Zhang, Y. F. Guo, D. Prabhakaran et al., Nat. Mater. 15, 27 (2016).

[13] B. Q. Lv, N. Xu, H. M. Weng, J. Z. Ma, P. Richard, X. C. Huang, L. X. Zhao, G. F. Chen, C. E. Matt, F. Bisti et al., Nat. Phys. 11, 724 (2015).

[14] B. Q. Lv, S. Muff, T. Qian, Z. D. Song, S. M. Nie, N. Xu, P. Richard, C. E. Matt, N. C. Plumb, L. X. Zhao et al., Phys. Rev. Lett. 115, 217601 (2015).

[15] A. A. Soluyanov, D. Gresch, Z. Wang, Q. Wu, M. Troyer, X. Dai, and B. A. Bernevig, Nature (London) 527, 495 (2015).

[16] Y. Sun, S.-C. Wu, M. N. Ali, C. Felser, and B. Yan, Phys. Rev. B 92, 161107 (2015).

[17] Z. Wang, D. Gresch, A. A. Soluyanov, W. Xie, S. Kushwaha, X. Dai, M. Troyer, R. J. Cava, and B. A. Bernevig, Phys. Rev. Lett. 117, 056805 (2016)

[18] T.-R. Chang, S.-Y. Xu, G. Chang, C.-C. Lee, S.-M. Huang, B. Wang, G. Bian, H. Zheng, D. S. Sanchez, I. Belopolski et al., Nat. Commun. 7, 10639 (2016).

[19] R. Clarke, E. Marseglia, and H. P. Hughes, Philos. Mag. B 38, 121 (1978).

[20] L. Huang, T. M. McCormick, M. Ochi, Z. Zhao, M.-T. Suzuki, R. Arita, Y. Wu, D. Mou, H. Cao, J. Yan et al., Nat. Mater. 15, 1155 (2016).

[21] A. Tamai, Q. S. Wu, I. Cucchi, F. Y. Bruno, S. Riccò, T. K. Kim, M. Hoesch, C. Barreteau, E. Giannini, C. Besnard et al., Phys. Rev. X 6, 031021 (2016).

[22] K. Deng, G. Wan, P. Deng, K. Zhang, S. Ding, E. Wang, M. Yan, H. Huang, H. Zhang, Z. Xu et al., Nat. Phys. 12, 1105 (2016).

[23] F. Y. Bruno, A. Tamai, Q. S. Wu, I. Cucchi, C. Barreteau, A. de la Torre, S. McKeown Walker, S. Riccò, Z. Wang, T. K. Kim et al., Phys. Rev. B 94, 121112 (2016).

[24] Y. Wu, D. Mou, N. H. Jo, K. Sun, L. Huang, S. L. Bud'ko, P. C. Canfield, and A. Kaminski, Phys. Rev. B 94, 121113 (2016).

[25] J. Sánchez-Barriga, M. G. Vergniory, D. Evtushinsky, I. Aguilera, A. Varykhalov, S. Blügel, and O. Rader, Phys. Rev. B 94, 161401 (2016).

[26] C. Wang, Y. Zhang, J. Huang, S. Nie, G. Liu, A. Liang, Y. Zhang, B. Shen, J. Liu, C. Hu et al., Phys. Rev. B 94, 241119 (2016).

[27] I. Belopolski, D. S. Sanchez, Y. Ishida, X. Pan, P. Yu, S.-Y. Xu, G. Chang, T.-R. Chang, H. Zheng, N. Alidoust et al., Nat. Commun. 7, 13643 (2016).

[28] H. Zheng, G. Bian, G. Chang, H. Lu, S.-Y. Xu, G. Wang, T.-R. Chang, S. Zhang, I. Belopolski, N. Alidoust et al., Phys. Rev. Lett. 117, 266804 (2016).

[29] Z. Huang, T. Das, A. V. Balatsky, and D. P. Arovas, Phys. Rev. B 87, 155123 (2013).

[30] T. Wehling, A. Black-Schaffer, and A. Balatsky, Adv. Phys. 63, 1 (2014).

[31] S. Heers, Ph.D. thesis, RWTH Aachen University, 2011.

[32] B. Zimmermann, P. Mavropoulos, N. H. Long, C.-R. Gerhorst, S. Blügel, and Y. Mokrousov, Phys. Rev. B 93, 144403 (2016).

[33] N. H. Long, P. Mavropoulos, B. Zimmermann, D. S. G. Bauer, S. Blügel, and Y. Mokrousov, Phys. Rev. B 90, 064406 (2014).

[34] R. Zeller, J. Phys. Condens. Matter 16, 6453 (2004).

[35] See Supplemental Material http://link.aps.org/supplemental/ 10.1103/PhysRevB.97.075106 for computational details and impurity resonance spectroscopy, which includes Refs. $[12,15,17,18,21,55-68]$.
[36] B. Feng, Y.-H. Chan, Y. Feng, R.-Y. Liu, M.-Y. Chou, K. Kuroda, K. Yaji, A. Harasawa, P. Moras, A. Barinov et al., Phys. Rev. B 94, 195134 (2016).

[37] P. Roushan, J. Seo, C. V. Parker, Y. S. Hor, D. Hsieh, D. Qian, A. Richardella, M. Z. Hasan, R. J. Cava, and A. Yazdani, Nature (London) 460, 1106 (2009).

[38] T. Zhang, P. Cheng, X. Chen, J.-F. Jia, X. Ma, K. He, L. Wang, H. Zhang, X. Dai, Z. Fang et al., Phys. Rev. Lett. 103, 266803 (2009).

[39] P. Sessi, F. Reis, T. Bathon, K. A. Kokh, O. E. Tereshchenko, and M. Bode, Nat. Commun. 5, 5349 (2014).

[40] I. Zeljkovic, Y. Okada, C.-Y. Huang, R. Sankar, D. Walkup, W. Zhou, M. Serbyn, F. Chou, W.-F. Tsai, H. Lin et al., Nat. Phys. 10, 572 (2014).

[41] S. Jeon, B. B. Zhou, A. Gyenis, B. E. Feldman, I. Kimchi, A. C. Potter, Q. D. Gibson, R. J. Cava, A. Vishwanath, and A. Yazdani, Nat. Mater. 13, 851 (2014).

[42] P. Sessi, Y. Sun, T. Bathon, F. Glott, Z. Li, H. Chen, L. Guo, X. Chen, M. Schmidt, C. Felser et al., Phys. Rev. B 95, 035114 (2017).

[43] P. Sessi, P. Rüßmann, T. Bathon, A. Barla, K. A. Kokh, O. E. Tereshchenko, K. Fauth, S. K. Mahatha, M. A. Valbuena, S. Godey et al., Phys. Rev. B 94, 075137 (2016).

[44] J. I. Pascual, G. Bihlmayer, Y. M. Koroteev, H.-P. Rust, G. Ceballos, M. Hansmann, K. Horn, E. V. Chulkov, S. Blügel, P. M. Echenique et al., Phys. Rev. Lett. 93, 196802 (2004).

[45] P. Sessi, R. R. Biswas, T. Bathon, O. Storz, S. Wilfert, A. Barla, K. A. Kokh, O. E. Tereshchenko, K. Fauth, M. Bode et al., Nat. Commun. 7, 12027 (2016).

[46] Z. E. A. Lin, 2D Mater. 3, 022002 (2016).

[47] J. Hong, Z. Hu, M. Probert, K. Li, D. Lv, X. Yang, L. Gu, N. Mao, Q. Feng, L. Xie et al., Nat. Commun. 6, 6293 (2015).

[48] H. Y. Jeong, S. Y. Lee, T. H. Ly, G. H. Han, H. Kim, H. Nam, Z. Jiong, B. G. Shin, S. J. Yun, J. Kim et al., ACS Nano 10, 770 (2016)

[49] V. Madhavan, W. Chen, T. Jamneala, M. F. Crommie, and N. S. Wingreen, Science 280, 567 (1998).

[50] A. K. Mitchell and L. Fritz, Phys. Rev. B 92, 121109 (2015).

[51] A. Narayanan, M. D. Watson, S. F. Blake, N. Bruyant, L. Drigo, Y. L. Chen, D. Prabhakaran, B. Yan, C. Felser, T. Kong et al., Phys. Rev. Lett. 114, 117201 (2015).

[52] Y. Li, Z. Wang, P. Li, X. Yang, Z. Shen, F. Sheng, X. Li, Y. Lu, Y. Zheng, and Z.-A. Xu, Front. Phys. 12, 127205 (2017).

[53] M. N. Ali, J. Xiong, S. Flynn, J. Tao, Q. D. Gibson, L. M. Schoop, T. Liang, N. Haldolaarachchige, M. Hirschberger, N. P. Ong et al., Nature (London) 514, 205 (2014).

[54] Y. Wang, E. Liu, H. Liu, Y. Pan, L. Zhang, J. Zeng, Y. Fu, M. Wang, K. Xu, Z. Huang et al., Nat. Commun. 7, 13142 (2016).

[55] Y. Qi, P. G. Naumov, M. N. Ali, C. R. Rajamathi, W. Schnelle, O. Barkalov, M. Hanfland, S.-C. Wu, C. Shekhar, Y. Sun et al., Nat. Commun. 7, 11038 (2016).

[56] A. Mar, S. Jobic, and J. A. Ibers, J. Am. Chem. Soc. 114, 8963 (1992).

[57] S. H. Vosko, L. Wilk, and M. Nusair, Can. J. Phys. 58, 1200 (1980).

[58] N. Stefanou, H. Akai, and R. Zeller, Comput. Phys. Commun. 60, 231 (1990).

[59] N. Stefanou and R. Zeller, J. Phys. Condens. Matter 3, 7599 (1991).

[60] FLEUR, http://www.flapw.de. 
[61] J. P. Perdew, K. Burke, and M. Ernzerhof, Phys. Rev. Lett. 77, 3865 (1996).

[62] I. Aguilera (private communication).

[63] D. S. G. Bauer, Ph.D. thesis, RWTH Aachen University, 2013.

[64] A. Aguayo, I. I. Mazin, and D. J. Singh, Phys. Rev. Lett. 92, 147201 (2004).
[65] K. Momma and F. Izumi, J. Appl. Crystallogr. 44, 1272 (2011).

[66] U. Ayachit, The ParaView Guide: A Parallel Visualization Application (Kitware Inc., New York, 2015).

[67] J. D. Hunter, Comput. Sci. Eng. 9, 90 (2007).

[68] B. E. Brown, Acta Crystallogr. 20, 268 (1966).

[69] W. Zhang, Q. Wu, and L. Zhang, Phys. Rev. B 96, 165125 (2017). 